\title{
A candidate accident tolerant fuel system based on a highly concentrated alloy thin film
}

\author{
M.A. Tunes ${ }^{\mathrm{a} *}$, V.M. Vishnyakova ${ }^{\mathrm{a}}$, O. Camara ${ }^{\mathrm{a}}$, G. Greaves ${ }^{\mathrm{a}}$, P.D. Edmondson ${ }^{\mathrm{b}}$, Y. Zhang ${ }^{\mathrm{b}}$ and S.E. Donnelly ${ }^{\mathrm{a}}$ \\ ${ }^{a}$ School of Computing and Engineering, University of Huddersfield, United Kingdom \\ ${ }^{b}$ Materials Sciences and Technology Division, Oak Ridge National Laboratory, United States of America
}

\begin{abstract}
The feasibility of depositing a thin film of highly concentrated alloy on zircaloy-4 substrates at low temperatures was investigated. Electron microscopy characterisation at meso- and nanoscales showed that the deposited thin film is equiatomic, single-phase and with all alloying elements uniformly distributed throughout the microstructure. Heavy-ion irradiations carried out in situ within a transmission electron microscope revealed the generation of both defect clusters and inert gas bubbles at around $1.5 \times 10^{16}$ ions $\cdot \mathrm{cm}^{-2}(15.4 \mathrm{dpa})$. Post-irradiation characterisation showed that the thin film preserved its solid solution and that, under the studied conditions, no elemental segregation or phase transformations were observed indicating a high radiation tolerance.
\end{abstract}

Keywords: Highly concentrated alloys; Accident Tolerant Fuels; Radiation Damage; Ion Beam Sputter-Deposition; Nuclear Energy

\section{Introduction}

Functional coatings have been applied to a wide variety of substrates aiming at increasing their applicability in different environments. Ceramic coatings, e.g. titanium nitride (TiN), are nowadays considered technological solutions to increase both reliability and life-time of mechanical tools and medical instruments because of improved anti-corrosion, chemical inertness and high hardness properties [1-3]. Metastable diamond-like-carbon (DLC) coatings are also applied to materials for demanding applications, such as automotive, aerospace and oil \& gas industries, because of its high hardness and low friction [4]. Applications of ceramics and metalloid coatings on optics and plasmonic components also open pathways to innovative technological functional devices $[5,6]$. Despite the relative success of the coating industries, problems such as low adhesion strength, poor tribological properties, incompatibility with metallic alloy substrates, nucleation and growth of voids and inert gas bubbles within the matrices during deposition are examples of recurrent challenges in the field for many applications [7-9].

Despite numerous existing applications of functional coatings in numerous areas of technology, utilisation of hard coatings within the nuclear field has been an area of not so focused intense research. Nevertheless, a particular application in this field involves the use of TiN and titanium aluminium nitride (TiAlN) coatings on zirconium alloys aiming to increase their oxidation resistance at high temperatures [10,11]. It is well-known that in the event of a loss-of-coolant accident (LOCA), a nuclear reactor core operating with $\mathrm{Zr}$-based alloy as the main nuclear fuel cladding material will generate large quantities of hydrogen due to the fast reaction kinetics of $\mathrm{Zr}$-steam oxidation [12,13]. The

\footnotetext{
*Corresponding authors: m.a.tunes@physics.org and v.vishnyakov@hud.ac.uk
} 
accumulation of hydrogen generated under these conditions was the primary cause of the FukushimaDaiichi nuclear disaster in Japan in 2011 which has motivated intense research efforts towards accident tolerant fuels (ATFs) $[14,15]$. The application of a hard protection coating barrier on Zr-based alloys is an alternative to address this problem and $\mathrm{Ti}(\mathrm{Al}) \mathrm{N}$ multilayer coatings are at the forefront of the most recent research and development efforts. However, a mandatory requirement for ATF materials is superior resistance and stability against the deleterious effects of energetic particle exposure. Recent work has shown that Ti-based coatings suffer from radiation-induced segregation (RIS) effects which create Ti-rich zones where oxidation reactions may occur similarly to those of $\mathrm{Zr}$ [16]. The assisted growth of inert gas bubbles via incorporation of segregated monoatomic $\mathrm{N}$ interstitial atoms was also identified which could limit the use of $\mathrm{Ti}(\mathrm{Al}) \mathrm{N}$ coatings on Zr-based alloys in the nuclear field [16]. MAX phases coatings are also under consideration for ATF systems [15], but in a recent work Wang et al. showed that the nucleation, growth and accumulation of inert gas bubbles at the interface with a Zr substrate contributed to decrease the adhesion after irradiation [17], indicating that the mitigation of displacement damage under irradiation is a key parameter in designing candidate materials for ATF systems.

Highly concentrated alloys (HCAs) - which recently have exhibited enormous potential to revolutionise future nuclear power plants [18-24] - are herein proposed as an alternative to ceramic coatings on Zr-based alloys [10,11]. HCAs have been reported to possess superior radiation tolerance against energetic particle irradiation resulting from their intrinsically tuneable chemical complexity and thus the atomic-level inhomogeneity and complex energy landscapes [18,25]. Some remarkable properties of HCAs include enhanced local recombination of irradiation-induced crystalline defects [18-20], higher potential barriers for atomic diffusion of solutes and defects [26] and high phase stability at high temperatures and under irradiation [27]. These core properties of HCAs are due to their complex crystalline structure $[18,20]$ which can be achieved by mixing alloying elements close to or at the equiatomic composition [28,29]. Despite their unique properties, the use of HCAs in the nuclear industry may be limited by cost and availability of alloying elements as well as the requirement of high-temperature processing. An alternative is the use of HCAs in the form of thin films [30-32].

The development of an accident tolerant fuel candidate system based on a highly concentrated alloy thin film (HCATF) is the main objective of this paper. For this, the feasibility of the HCATF on a zircaloy-4 substrate at low temperatures is firstly investigated. Cross-sectional TEM lamellae of the HCATF deposited on zircaloy-4 were then subjected to heavy-ion irradiation in situ within a TEM up to $15.4 \mathrm{dpa}$ (displacements per atom) at $573 \mathrm{~K}$ to analyse the radiation response of the system. Under the studied conditions, post-irradiation analytical characterisation indicated that the thin film retained its phase without noticeable elemental segregation, although inert gas bubbles and black-spot damage were observed to form throughout the microstructure. Interfacial effects (e.g., delamination and exfoliation) were not observed. The results indicate a superior radiation tolerance of the HCATF when compared with similar heavy-ion irradiations previously reported for ceramic coatings [16].

\section{Materials and Methods}

Prior to deposition, zircaloy-4 specimens were electropolished using an electrolyte solution of $90 \%$ perchloric acid $\left(\mathrm{HClO}_{4}\right)$ and $10 \%$ methanol $\left(\mathrm{CH}_{3} \mathrm{OH}\right)$ at a temperature of $233 \mathrm{~K}$ with an electric potential of $20 \mathrm{~V}$ for 2 minutes. After electropolishing, the samples were washed several times in a pure methanol bath and were left to dry in air. Zircaloy- 4 substrates were inserted into an ion-beam 
sputter-deposition chamber and equiatomic thin films of quaternary FeCrMnNi were deposited onto substrates from four elemental targets for two hours. The procedure to achieve the desired equiatomic composition using the IBSD technique was described in a previous paper [31]. The substrate temperature was monitored during the deposition and was measured to be in the range of $350-370 \mathrm{~K}$ and the deposition pressure in the deposition chamber was around of $3 \times 10^{-2} \mathrm{~Pa}$. The chamber base pressure was at around $1 \times 10^{-4} \mathrm{~Pa}$

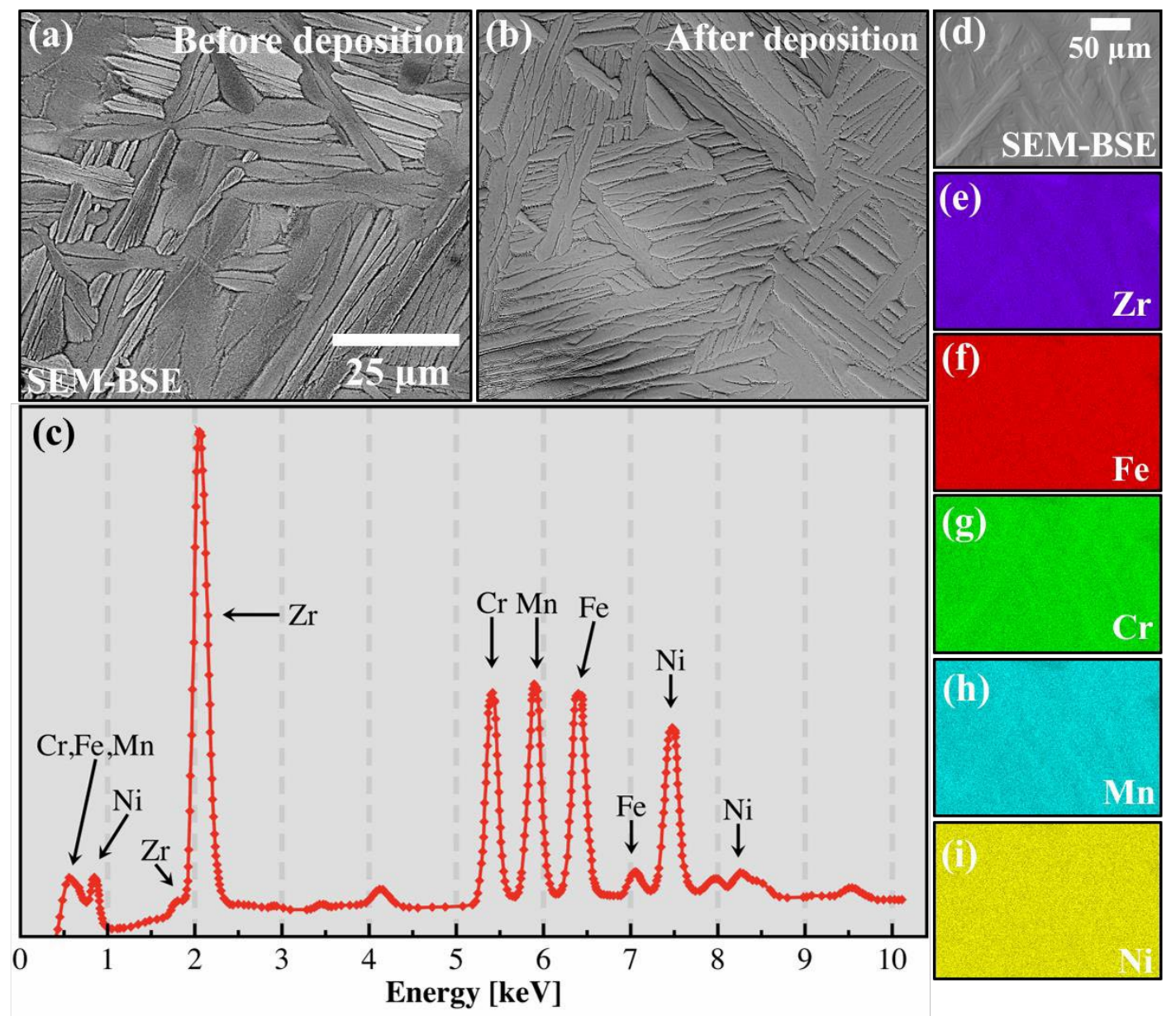

Figure 1: SEM-BSE characterisation of the zircaloy-4 hexagonal microstructure (a) prior and (b) post deposition. The EDX spectrum of the FeCrMnNi HCATF is shown in (c). Images from (e) to (i) exhibit an elemental characterisation of the HCATF/zircaloy-4 system. Note: the scale bar in (a) also applies to (b) and the scale bar in (d) applies to (e-i).

Electron microscopy characterisation at mesoscale was performed with a FEI VERSA 3D Dual Beam Field-Emission Gun (FEG) scanning electron microscope (SEM) equipped with an Oxford Instruments Energy Dispersive X-ray (EDX) spectrometer. Elemental quantification of the characteristic X-rays was performed with the Cliff-Lorimer standardless method and the EDX system was calibrated with stoichiometric $\mathrm{SiC}$ and pure Mo reference samples. TEM samples were produced using conventional FIB lift-out technique. At the nanoscale, the characterisation has been performed with a JEOL JEM 2100F operating at $200 \mathrm{kV}$ with a FEG and with a Hitachi H-9500 TEM operating at $300 \mathrm{kV}$ with a $\mathrm{LaB}_{6}$ filament.

Medium-energy heavy-ion irradiation in situ within a TEM was carried out in the crosssectional lamellae using the MIAMI-2 system with $134 \mathrm{keV} \mathrm{Xe}^{+}$ions at $573 \mathrm{~K}$ with a flux of $1.4 \times 10^{13}$ 
ions $\cdot \mathrm{cm}^{-2} \cdot \mathrm{s}^{-1}$. Under these conditions, both the implantation depth and the damage profiles are nearly uniform across the thin foil (thickness of around $70 \mathrm{~nm}$ ). The irradiation temperature was chosen to represent the operational conditions of light-water reactors (LWRs), and the ion species and energy were selected due to the suggestions proposed by English et al. on the use of low-to-medium energy heavy ion irradiation to simulate the displacement cascades in neutron irradiation environments [33]. Fluence-to-dpa conversion was performed following a procedure suggested by Stoller et al. using the outputs of the Monte Carlo code SRIM-2013 [34]. The TEM lamellae were irradiated up to a maximum fluence of $1.5 \times 10^{16}$ ions $\mathrm{cm}^{-2}$ corresponding to $15.4 \mathrm{dpa}$ for the thin film. Post-irradiation Energy Filtered TEM (EFTEM) characterisation was performed using the H-9500. Fresnel contrast was used to characterise the presence of voids and bubbles before, during and after irradiation.

\section{Results}

The microstructure at the mesoscale of the zircaloy-4 substrate after electropolishing and prior to deposition is shown in the backscattered electron (SEM-BSE) micrograph in figure 1(a). After the deposition, the microstructure of the FeCrMnNi HCATF on the zircaloy-4 is shown in the micrograph in figure 1(b) and its corresponding EDX spectrum as well as the elemental mappings are shown in figures 1(c) and 1(d-i), respectively. The EDX elemental quantification of the substrate before and HCATF after deposition is listed in table 1.

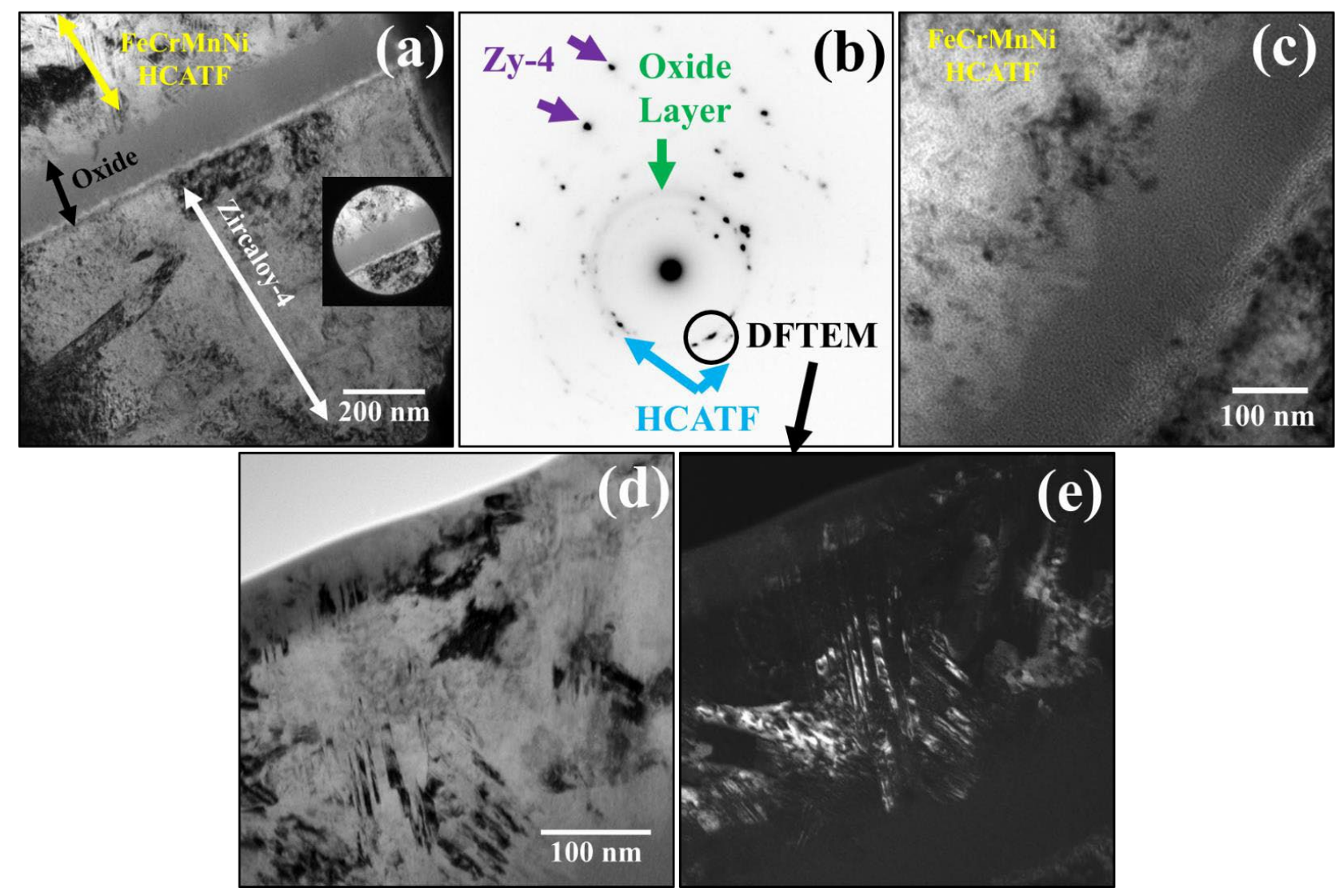

Figure 2: The microstructure of the HCATF/zircaloy-4 system at nanoscale is exhibited in (a). The diffraction pattern (b) was taken as indicated by the inset in (a). The overfocused BFTEM micrograph (c) exhibit the presence of voids in the oxide layer and in the HCATF. The micrographs (d) and (e) show the microstructure of the HCATF at a higher magnification in bright- and dark-field, respectively. 
Table 1: The elemental compositions from the EDX analysis of the zircaloy-4 substrate before deposition and after electropolishing and of the HCATF after deposition (the uncertainty is within $2 \%$ ).

\begin{tabular}{|c|c|c|}
\hline Element & Substrate [wt\%] & HCATF [at\%] \\
\hline $\mathbf{Z r}$ & 95.3 & - \\
\hline $\mathbf{S n}$ & 1.2 & - \\
\hline $\mathbf{F e}$ & - & 25.3 \\
\hline $\mathbf{C r}$ & - & 23.4 \\
\hline $\mathbf{M n}$ & - & 24.3 \\
\hline $\mathbf{N i}$ & - & 27.0 \\
\hline $\mathbf{O}$ & 3.5 & - \\
\hline
\end{tabular}

The cross-sectional profile of the HCATF and the zircaloy-4 substrate is shown in the BrightField TEM (BFTEM) micrograph in figure 2(a). A zirconium oxide layer of $\sim 100 \mathrm{~nm}$ was observed between the substrate and the deposited thin film. After deposition, O X-ray signal is not detected in the EDX analysis due to absorption by the film. The diffraction pattern in figure 2(b) was taken using the selected-area diffraction aperture as indicated by the inset in figure 2(a). By defocusing the electron beam ( $1 \mu \mathrm{m}$ degree of underfocus), cavities of 1-2 $\mathrm{nm}$ in diameter have been observed primarily concentrated in the oxide layer, as revealed by the speckled contrast in figure 2(c). These cavities have also been observed in the HCATF as exhibited in figures 2(c) and 4(a). The underfocused BF and DFTEM (at focus) micrographs in figures 2(d) and 2(f) show the microstructure of the HCATF at a higher magnification revealing the presence of planar defects (twins) which have recently been associated with high cracking resistance in similar HCAs [29].

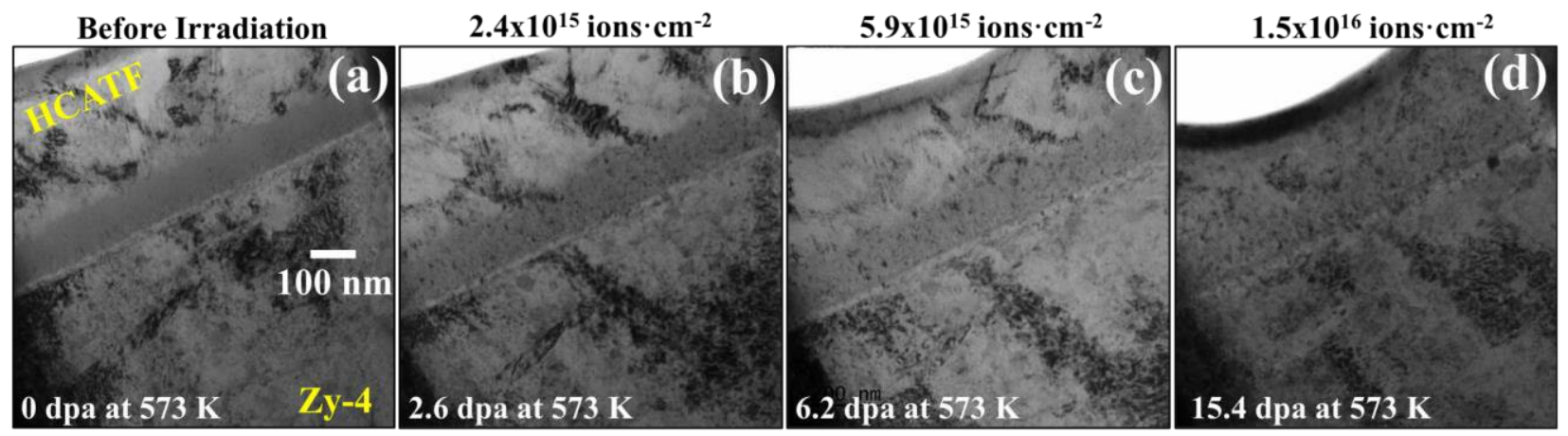

Figure 3: Microstructural evolution of the HCATF, the zirconium oxide layer and the zircaloy-4 substrate during heavy-ion irradiation. Note: the scale bar in the micrograph (a) applies to all micrographs in the figure.

Following the electron microscopy characterisation at the meso- and nanoscales, the crosssectional electron-transparent lamellae were irradiated with $134 \mathrm{keV} \mathrm{Xe}$ ions in situ within the TEM. Figure 3 shows a set of BFTEM micrographs taken before and during the irradiation. With increasing ion fluence, a small degree of edge sputtering and bending was observed to occur. Black-spot (interstitial or vacancy clusters) formed in the substrate, in the HCATF and in the oxide layer microstructures. At around $6.2 \mathrm{dpa}\left(5.9 \times 10^{15}\right.$ ions $\left.\cdot \mathrm{cm}^{-2}\right)$, Xe bubbles were observed in the HCATF and in the oxide layer and a comparison between the pre-existing cavities and the Xe bubbles after irradiation is exhibited in figure 4. Under such studied conditions, post-irradiation characterisation with EFTEM revealed that the HCATF had its random solid solution preserved with no detectable elemental segregation or formation of secondary phases as shown in figure 5. At the end of the experiment, the thickness of the oxide layer is reduced. 

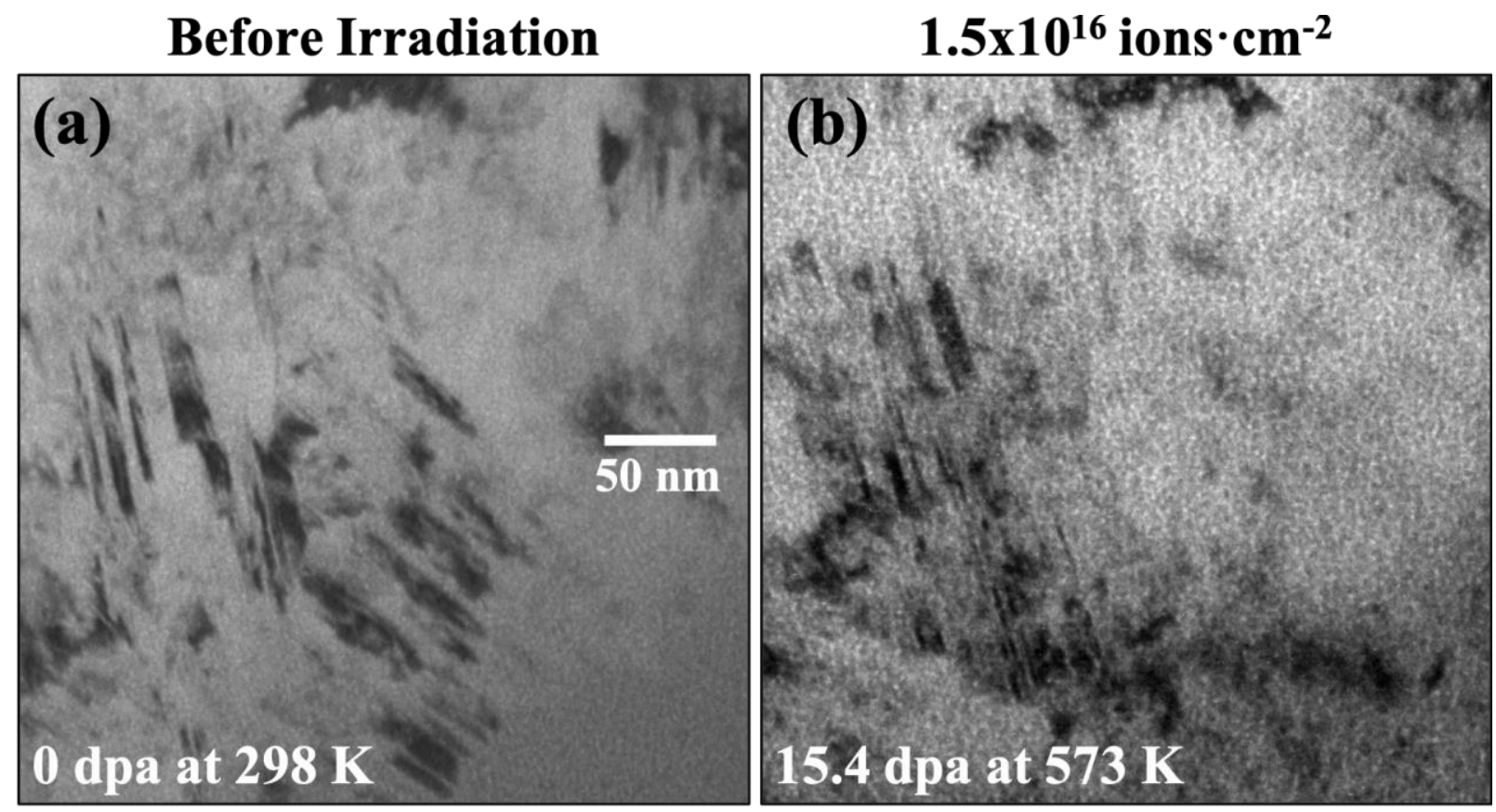

Figure 4: Underfocused BFTEM micrographs of the HCATF microstructure (a) before irradiation and (b) after irradiation to $15.4 \mathrm{dpa}$. Note: scale bar in (a) also applies to (b) and the micrographs were taken at a defocus degree of $500 \mathrm{~nm}$.

\section{Discussion}

From the EDX analysis, a small amount of oxygen was detected in the zircaloy-4 substrate after electropolishing. Oxide layers (passivation) can readily form on freshly-electropolished metallic surfaces of metals like $\mathrm{Zr}$ and $\mathrm{Ti}$ due to the large negative energy of formation: approximately -1000 $\mathrm{kJ} \cdot \mathrm{mol}^{-1}$ for $\mathrm{Zr}$ [35]. As a result, a porous oxide layer was observed in the conventional BFTEM analysis of the cross-sectional lamella and its thickness was measured to be approximately $150 \mathrm{~nm}$. The formation of a self-passivating layer formed during electropolishing is a well-known phenomenon that protects the zircaloy-4 from further oxidation. Prior to deposition, the microstructure of the zircaloy-4 substrate exhibited needle-like grains that are characteristic of a Widmanstätten pattern. The microstructural grain-morphology of the zircaloy-4 substrate was not changed post deposition of the thin-film as can be seen in figure 1b. As revealed by the SEM-EDX elemental maps, the alloying elements of the HCATF were uniformly distributed throughout the substrate and point EDX elemental quantification confirmed its equiatomic elemental composition. Under these conditions, the FeCrMnNi HCATF was characterised to have face-centred cubic crystal structure as shown previously [31].

Temperature and working pressure are parameters that dictate, in many cases, the final microstructure of a plasma vapour deposited (PVD) thin film according to the zone models developed by Movchan-Demichishin-Thornton (MDT) [7]. The melting temperature $\left(\mathrm{T}_{\mathrm{m}}\right)$ of the equiatomic system FeCrMnNi can be assumed to be within the range of 1700-1800 K which is typical of ferrous alloys with $\mathrm{Cr}, \mathrm{Mn}$ and $\mathrm{Ni}$ as major alloying elements. Herein, given the experimental conditions, the temperature for the HCATF deposition on the zircaloy-4 substrate was approximately $0.2 \mathrm{~T}_{\mathrm{m}}$. MDT zone models assume that for low temperature deposition (less than $0.3 \mathrm{~T}_{\mathrm{m}}$ ) the typical thin film final microstructure at the nanoscale consists of columnar grains and voided grain boundaries which is not the case for the HCATF studied in this work. Such microstructural differences between equiatomic 
and conventional thin films are attributed to the characteristic thermodynamics of highly concentrated solid solution alloys: the lower Gibbs free energy of these systems, which is achieved via maximising the entropy at (or close to) equiatomic conditions, enhances the kinetics of embryo nucleation promoting the faster growth of larger grains when compared with non-equiatomic thin film systems [31]. Under such conditions, the final microstructure of the HCATF does not exhibit phase separation, precipitation or voided grain boundaries.

Speckled Fresnel contrast was observed in the oxide layer and in the HCATF regions close to the interface by defocusing the electron beam. This indicates that surface roughening and/or cavities (voids or Ar bubbles) have formed as result of the deposition. The synthesis of thin films via the PVD processes can be interpreted as involving a low energy ion bombardment and some ion implantation mechanisms in which the reduced elemental mobility at temperatures of around $0.2 \mathrm{~T}_{\mathrm{m}}$ promotes clustering of implanted interstitials species, including Ar (from the plasma). The occurrence of such defects has been the subject of intense past research mainly by Lloyd [36] and Nakahara [37], and still remains a challenge in the deposition of thin solid films.
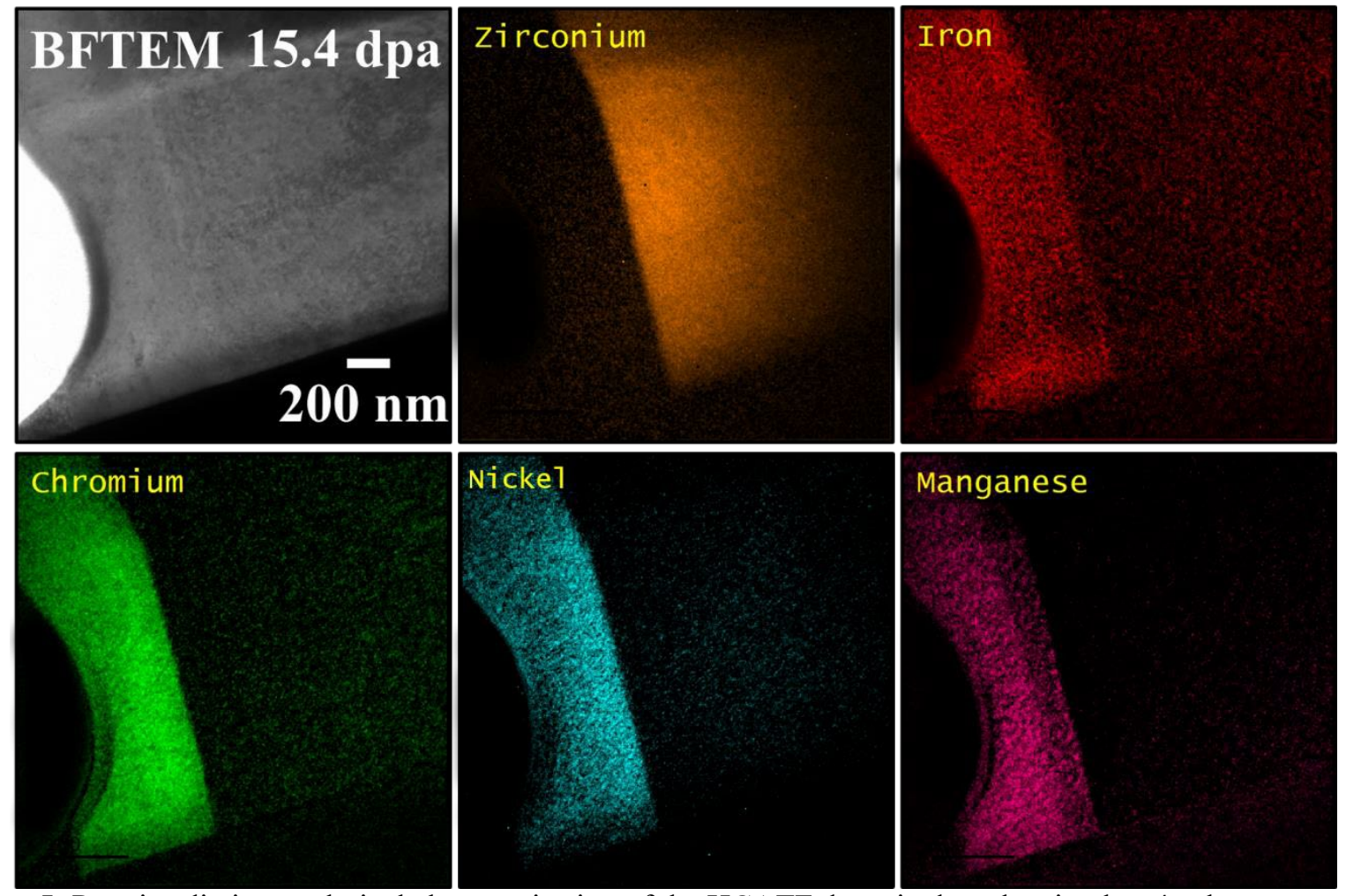

Figure 5: Post-irradiation analytical characterisation of the HCATF deposited on the zircaloy-4 substrate.

The radiation response of the HCATF deposited on zircaloy-4 exhibited interesting results. Formation of black-spots was observed in the oxide layer at doses of 2.6-6.2 dpa whilst in the HCATF, this type of defect as only observed at $15.4 \mathrm{dpa}$ (the maximum dose in this study). Xe nanobubbles became observable at approximately $6.2 \mathrm{dpa}$ and did not grow significantly up to the end of the experiment. Upon increasing dose, the oxide layer was observed to decrease in size (shrink in thickness without any observable cracking) and, at $15.4 \mathrm{dpa}$, the interface between the HCATF and the oxide layer was no longer observable suggesting that it has been partially mixed under irradiation, although without affecting the adhesion of the deposited thin film with the substrate. The pre-existence of voids 
in the oxide layer as shown in the micrograph 2(c) and their subsequent annihilation during irradiation are factors that could contribute to such dissolution. EFTEM qualitative characterisation of the deposited film has indicated that no significant radiation-induced elemental segregation or precipitation occurred, and the random solid solution was preserved up to the maximum dose of this work in contrast with similar in situ TEM ion irradiations for TiN coatings reported previously [16]. It is worth emphasising that low signal-to-noise ratios of $\mathrm{Ni}, \mathrm{Cr}$ and $\mathrm{Fe}$ were detected in the zircaloy4 substrate, but this rather due to the fact they are minor alloying elements in this specific alloy than segregated atoms diffusing from the substrate during irradiation. The conventional and analytical characterisations presented in this work are indicative that the HCATF possess a certain degree of radiation resistance and it has the potential to be a part of future ATF systems.

The high-phase stability of highly concentrated alloys under energetic particle irradiation has been attributed to their intrinsic (different) defect dynamics. The chemical disorder promoted by the equiatomic mixing is responsible for suppressing atomic diffusion, enhancing defect recombination, and delaying the growth of extended damage structures. The observed improvement of radiation resistance is attributed to energy dissipation that, on increasing chemical complexity from pure metals to multiple-element HCAs, has been demonstrated to induce an orders-of-magnitude reduction in electron mean free paths and electron and thermal conductivities that significantly suppress damage accumulation under ion irradiation $[19,20,26]$. Due to these facts, HCATFs are here proposed as new pathways towards the development of ATF alloys with enhanced radiation tolerance.

\section{Conclusions}

Materials for highly demanding applications are usually synthesised at high temperatures aiming at achieving certain microstructures with high thermodynamic stability. In some cases, the full thermodynamic equilibrium is not achieved due to diffusional problems during cooling or quenching. For nuclear energy applications, materials or alloys that are not thermodynamically stable can suffer from gradual phase transformations and damage accumulation that will limit their applicability and lifetime, and in extreme cases, lead to severe accidents. In this work, the feasibility of the deposition - at low homologous temperatures - of a highly concentrated alloy thin film on a zircaloy-4 substrate has been investigated and its radiation response to heavy ion irradiation was studied in situ within a TEM at $573 \mathrm{~K}$ to simulate the standard operating conditions of a light-water reactor. Displacement damage generation in the HCATF has been observed to occur at high doses $(\sim 12 \mathrm{dpa})$, and the formation of Xe nanobubbles has been observed at lower doses, around $6 \mathrm{dpa}$; these did not grow significantly with increasing dose. Radiation induced segregation, precipitation, amorphisation and phase separation have not been observed. The HCATF has its random solid solution nature preserved at the end of the irradiation experiments indicating a superior phase stability under energetic particle exposure. The oxide layer observed between the HCATF and the zircaloy-4 substrate was subjected to irradiation-assisted dissolution with no impact on the thin film adhesion to the substrate surface. Therefore, if coating zirconium alloys is considered a prominent solution to creating accident tolerant fuels, highly concentrated alloy thin films are herein proposed as an alternative candidate to conventional ceramics, such as TiN in which heavy ion irradiations at similar conditions have shown an extensive range of radiation-induced defects including phase instabilities. Further studies to assess the HCATFs' microstructural evolution at higher temperatures and doses are necessary to determine their consideration for innovative nuclear reactor concepts (generations III+ and IV). Ex situ ion irradiation studies with post-irradiation characterisation including X-ray diffraction (XRD) as well as 
nanomechanical measurements are also needed to address the phase stability and tribological properties of the HCATFs.

\section{Acknowledgment:}

MAT was supported through the ASTRO fellowship, a United States Department of Energy workforce development program implemented at Oak Ridge National Laboratory through the Oak Ridge Institute for Science and Education under contract DE-AC05-06OR23100. YZ was supported as part of the Energy Dissipation to Defect Evolution (EDDE), an Energy Frontier Research Center funded by the US Department of Energy, Office of Science, Basic Energy Sciences under contract number DEAC05-00OR22725. PDE acknowledges funding from the US Department of Energy, Office of Fusion Energy Science. We would also like to acknowledge the United Kingdom Engineering and Physical Sciences Research Council (EPSRC) for funding the MIAMI-2 system under grant EP/M028283/1.

\section{Declaration of Interest Statement}

The authors declare no conflict of interest.

\section{Data availability}

The processed/raw data required to reproduce these findings are available to download using the link doi:10.17632/2nm52sxb7k.3 from Mendeley Data.

\section{References}

[1] I. Konyashin and G. Fox-Rabinovich, Nanograined titanium nitride thin films, Adv. Mater. 10 (1998), pp. 952-955.

[2] W. Münz, Titanium aluminum nitride films: A new alternative to TiN coatings, J. Vac. Sci. Technol. A Vacuum, Surfaces, Film. 4 (1986), pp. 2717-2725.

[3] C.Y.H. Lim, S.C. Lim and K.S. Lee, Performance of TiN-coated high speed steel tool inserts in turning, Tribol. Int. 32 (1999), pp. 393-398.

[4] B.D. Beake, T.W. Liskiewicz, V.M. Vishnyakov and M.I. Davies, Development of DLC coating architectures for demanding functional surface applications through nano- and micro-mechanical testing, Surf. Coatings Technol. 284 (2015), pp. 334-343.

[5] J.A. Huang and L.B. Luo, Low-Dimensional Plasmonic Photodetectors: Recent Progress and Future Opportunities, Adv. Opt. Mater. 6 (2018), pp. 1701282.

[6] D. Shah, A. Catellani, H. Reddy, N. Kinsey, V. Shalaev, A. Boltasseva et al., Controlling the Plasmonic Properties of Ultrathin TiN Films at the Atomic Level, ACS Photonics 5 (2018), pp. 2816-2824.

[7] J.A. Thornton, The microstructure of sputter-deposited coatings, J. Vac. Sci. Technol. A Vacuum, Surfaces, Film. 4 (1986), pp. 3059-3065.

[8] J.A. Thornton, High Rate Thick Film Growth, Annu. Rev. Mater. Sci. 7 (1977), pp. 239-260.

[9] A. Matthews and A.R. Lefkow, Problems in the physical vapour deposition of titanium nitride, Thin Solid Films 126 (1985), pp. 283-291.

[10] E. Alat, A.T. Motta, R.J. Comstock, J.M. Partezana and D.E. Wolfe, Ceramic coating for corrosion (c3) resistance of nuclear fuel cladding, Surf. Coatings Technol. 281 (2015), pp. $133-143$.

[11] E. Alat, A.T. Motta, R.J. Comstock, J.M. Partezana and D.E. Wolfe, Multilayer (TiN, TiAlN) ceramic coatings for nuclear fuel cladding, J. Nucl. Mater. 478 (2016), pp. 236-244. 
[12] I. Younker and M. Fratoni, Neutronic evaluation of coating and cladding materials for accident tolerant fuels, Prog. Nucl. Energy 88 (2016), .

[13] K.A. Terrani, S.J. Zinkle and L.L. Snead, Advanced oxidation-resistant iron-based alloys for LWR fuel cladding, J. Nucl. Mater. 448 (2014), pp. 420-435.

[14] S.J. Zinkle, K.A. Terrani, J.C. Gehin, L.J. Ott and L.L. Snead, Accident tolerant fuels for LWRs: A perspective, J. Nucl. Mater. 448 (2014), pp. 374-379.

[15] Accident tolerant fuel cladding development: Promise, status, and challenges. North-Holland, 2018.

[16] M.A. Tunes, F.C. da Silva, O. Camara, C.G. Schön, J.C. Sagás, L.C. Fontana et al., Energetic particle irradiation study of TiN coatings: are these films appropriate for accident tolerant fuels?, J. Nucl. Mater. 512 (2018), pp. 239-245.

[17] J. Wang, R. Shu, Y. Dong, T. Shao, Q.H. Deng, X.B. Zhou et al., Microstructure evolution of V2AlC coating on Zr substrate under He irradiation and their mechanical behavior, Scr. Mater. 137 (2017), pp. 13-17.

[18] Y. Zhang, K. Jin, H. Xue, C. Lu, R.J. Olsen, L.K. Beland et al., Influence of chemical disorder on energy dissipation and defect evolution in advanced alloys, J. Mater. Res. 31 (2016), pp. 2363-2375.

[19] C. Lu, L. Niu, N. Chen, K. Jin, T. Yang, P. Xiu et al., Enhancing radiation tolerance by controlling defect mobility and migration pathways in multicomponent single-phase alloys, Nat. Commun. 7 (2016), pp. 13564.

[20] Y. Zhang, G.M. Stocks, K. Jin, C. Lu, H. Bei, B.C. Sales et al., Influence of chemical disorder on energy dissipation and defect evolution in concentrated solid solution alloys, Nat. Commun. 6 (2015), pp. 8736.

[21] C. Lu, T. Yang, K. Jin, G. Velisa, P. Xiu, M. Song et al., Enhanced void swelling in NiCoFeCrPd high-entropy alloy by indentation-induced dislocations, Mater. Res. Lett. 6 (2018), pp. 584-591.

[22] F. Zhang, Y. Tong, K. Jin, H. Bei, W.J. Weber, A. Huq et al., Chemical complexity induced local structural distortion in $\mathrm{NiCoFeMnCr}$ high-entropy alloy, Mater. Res. Lett. 6 (2018), pp. 450-455.

[23] K.M. Youssef, A.J. Zaddach, C. Niu, D.L. Irving and C.C. Koch, A novel low-density, highhardness, high-entropy alloy with close-packed single-phase nanocrystalline structures, Mater. Res. Lett. 3 (2014), pp. 95-99.

[24] M.-H. Tsai and J.-W. Yeh, High-Entropy Alloys: A Critical Review, Mater. Res. Lett. 2 (2014), pp. 107-123.

[25] C.G. Schön, T. Duong, Y. Wang and R. Arróyave, Probing the entropy hypothesis in highly concentrated alloys, Acta Mater. 148 (2018), pp. 263-279.

[26] S. Zhao, W.J. Weber and Y. Zhang, Unique Challenges for Modeling Defect Dynamics in Concentrated Solid-Solution Alloys, JOM 69 (2017), pp. 2084-2091.

[27] F. Granberg, K. Nordlund, M.W. Ullah, K. Jin, C. Lu, H. Bei et al., Mechanism of radiation damage reduction in equiatomic multicomponent single phase alloys, Phys. Rev. Lett. 116 (2016), pp. 135504.

[28] B.S. Murty, J.-W. Yeh and S. Ranganathan, High-Entropy Alloys, Butterworth-Heinemann, 2014.

[29] Z.J. Zhang, M.M. Mao, J. Wang, B. Gludovatz, Z. Zhang, S.X. Mao et al., Nanoscale origins of the damage tolerance of the high-entropy alloy CrMnFeCoNi, Nat. Commun. 6 (2015), pp. 10143.

[30] T. Egami, W. Guo, P.D. Rack and T. Nagase, Irradiation Resistance of Multicomponent Alloys, Metall. Mater. Trans. A 45 (2014), pp. 180-183.

[31] M.A. Tunes, V.M. Vishnyakov and S.E. Donnelly, Synthesis and characterisation of highentropy alloy thin films as candidates for coating nuclear fuel cladding alloys, Thin Solid 
Films 649 (2018), pp. 115-120.

[32] Z. An, H. Jia, Y. Wu, P.D. Rack, A.D. Patchen, Y. Liu et al., Solid-Solution CrCoCuFeNi High-Entropy Alloy Thin Films Synthesized by Sputter Deposition, Mater. Res. Lett. 3 (2015), pp. 203-209.

[33] C.A. English and B.L. Eyre, Heavy-ion damage in Alpha-Fe, Nature 260 (1976), pp. 619-21.

[34] R.E. Stoller, M.B. Toloczko, G.S. Was, A.G. Certain, S. Dwaraknath and F.A. Garner, On the use of SRIM for computing radiation damage exposure, Nucl. Instruments Methods Phys. Res. Sect. B Beam Interact. with Mater. Atoms 310 (2013), pp. 75-80.

[35] M.F. Ashby and D.R.H. Jones, Engineering Materials, Elsevier/Butterworth-Heinemann, 2013.

[36] J.R. Lloyd and S. Nakahara, Voids in thin as deposited gold films prepared by vapor deposition, J. Vac. Sci. Technol. 14 (1977), pp. 655-659.

[37] S. Nakahara, Microporosity induced by nucleation and growth processes in crystalline and non-crystalline films, Thin Solid Films 45 (1977), pp. 421-432. 


\section{List of Tables}

Table 2: The elemental compositions from the EDX analysis of the zircaloy-4 substrate before deposition and after electropolishing and of the HCATF after deposition (the uncertainty is within $2 \%)$. 4

\section{List of Figures}

Figure 1: SEM-BSE characterisation of the zircaloy-4 hexagonal microstructure (a) prior and (b) post deposition. The EDX spectrum of the FeCrMnNi HCATF is shown in (c). Images from (e) to (i) exhibit an elemental characterisation of the HCATF/zircaloy-4 system. Note: the scale bar in (a) also applies to (b) and the scale bar in (d) applies to (e-i).

Figure 2: The microstructure of the HCATF/zircaloy-4 system at nanoscale is exhibited in (a). The diffraction pattern (b) was taken as indicated by the inset in (a). The overfocused BFTEM micrograph (c) exhibit the presence of voids in the oxide layer and in the HCATF. The micrographs (d) and (e) show the microstructure of the HCATF at a higher magnification in bright- and dark-field, respectively

Figure 3: Microstructural evolution of the HCATF, the zirconium oxide layer and the zircaloy-4 substrate during heavy-ion irradiation. Note: the scale bar in the micrograph (a) applies to all micrographs in the figure

Figure 4: Underfocused BFTEM micrographs of the HCATF microstructure (a) before irradiation and (b) after irradiation to 15.4 dpa. Note: scale bar in (a) also applies to (b) and the micrographs were taken at a defocus degree of $500 \mathrm{~nm}$.

Figure 5: Post-irradiation analytical characterisation of the HCATF deposited on the zircaloy-4 substrate. 


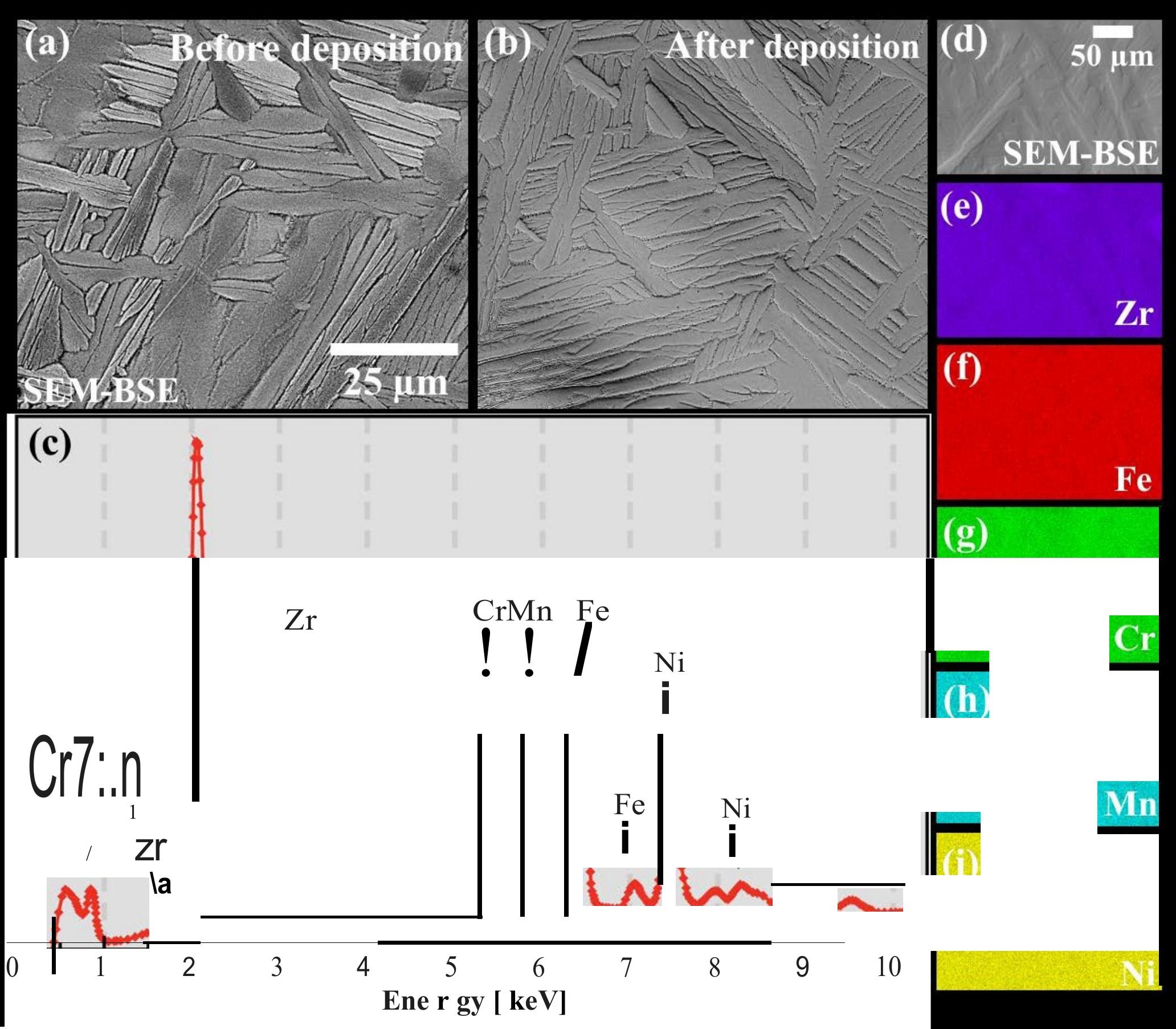




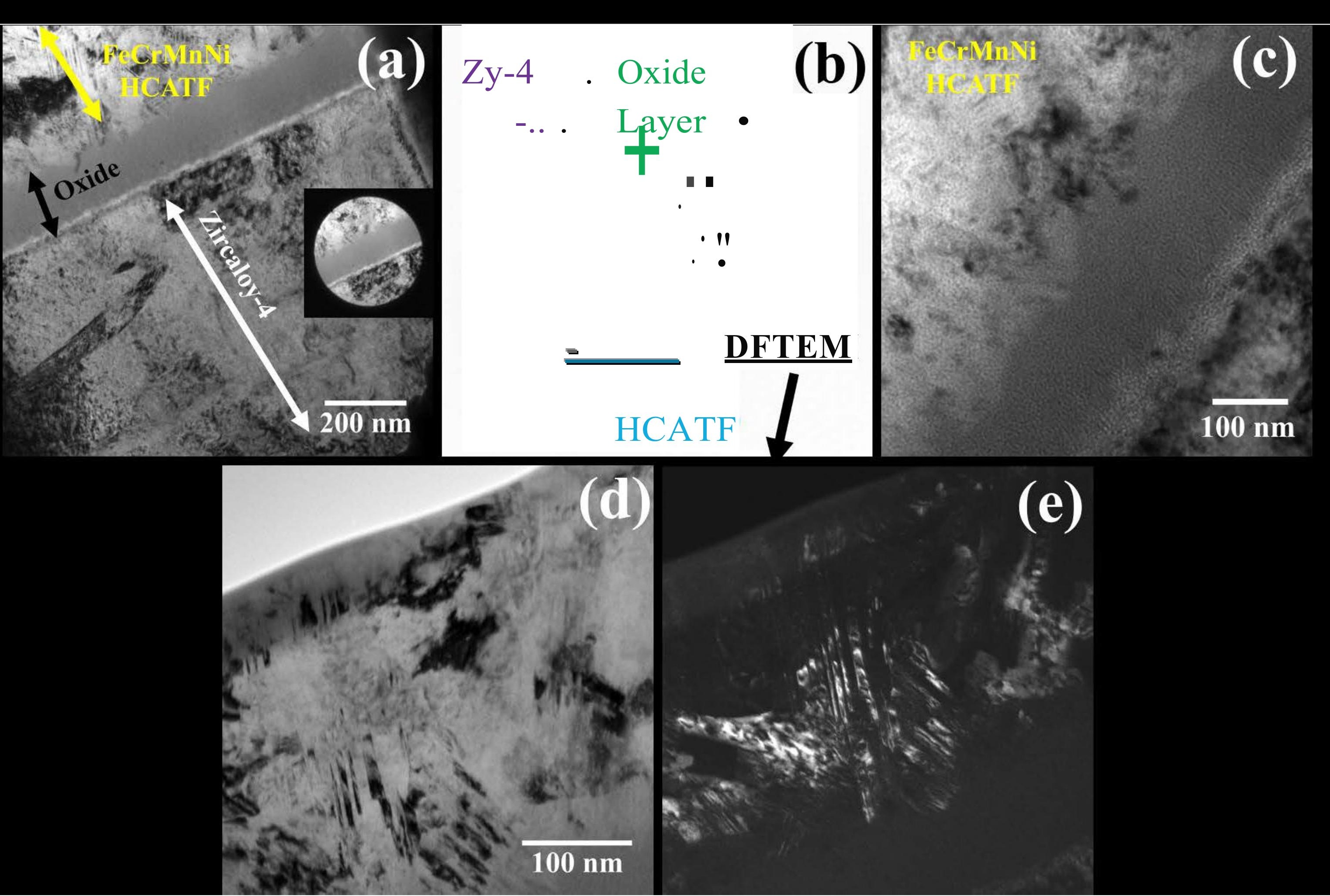


(a)

0 dpa at $298 \mathrm{~K}$

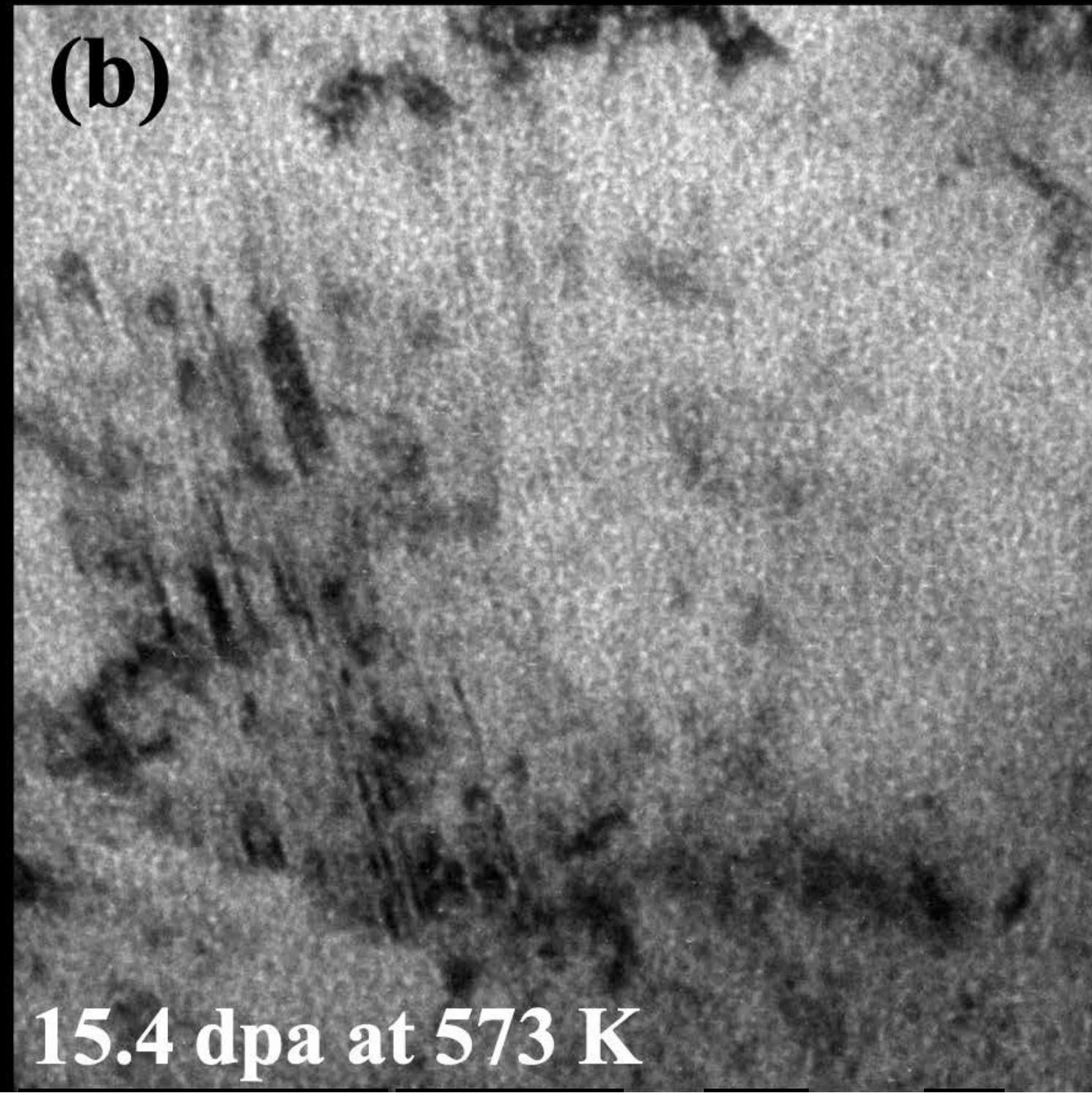




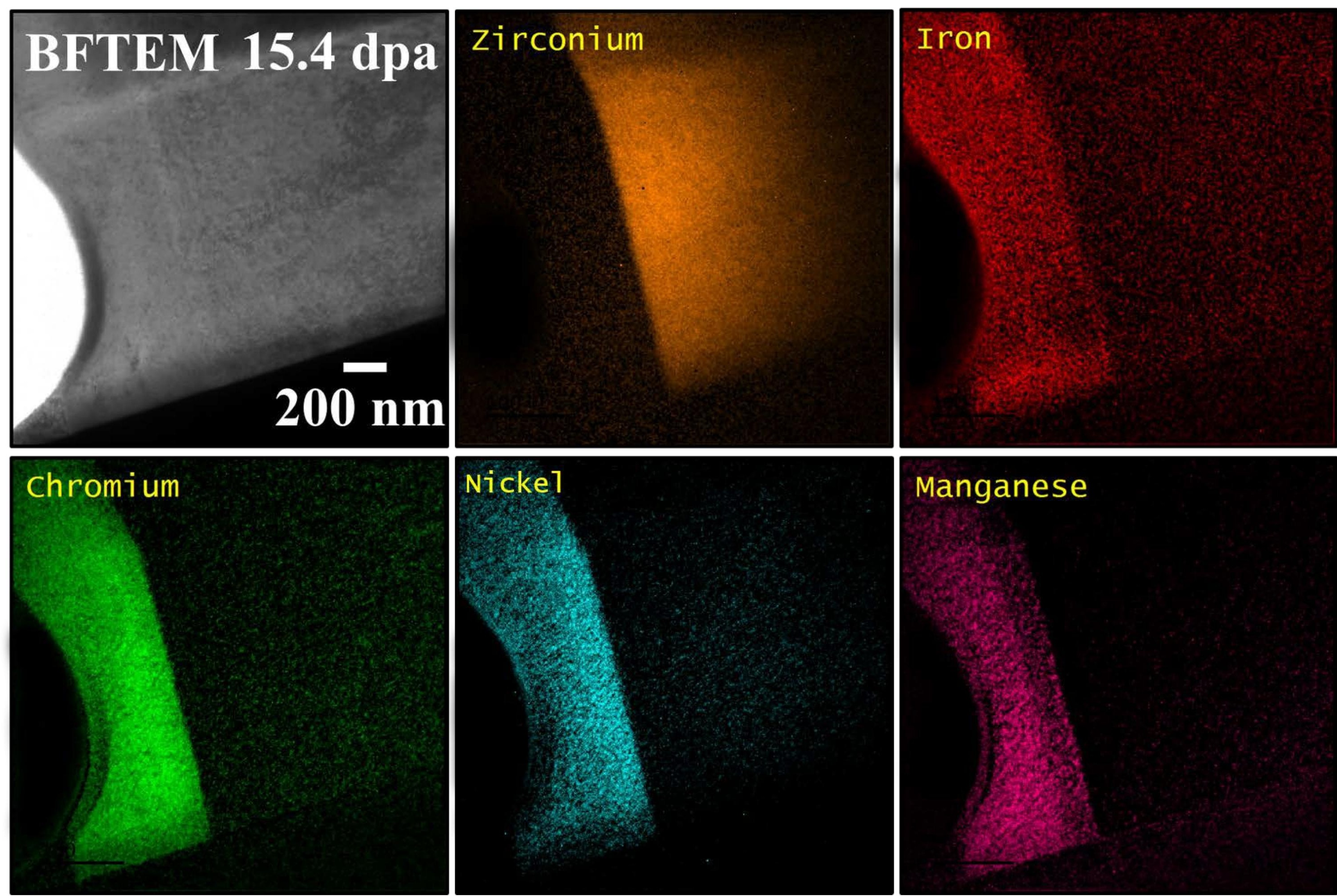

